\title{
A CMOS MONOLITHICALLY-INTEGRATED THREE-AXIS ACCELEROMETER BASED ON THERMAL CONVECTION
}

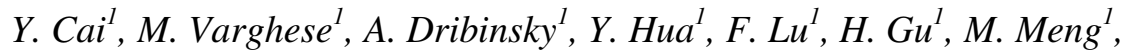

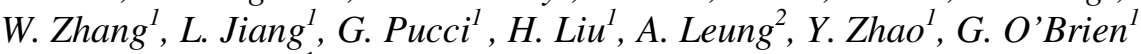 \\ ${ }^{1}$ Memsic Inc., Andover, MA, USA \\ ${ }^{2}$ Simon Fraser University, Burnaby, BC, Canada
}

\begin{abstract}
We report on the design, fabrication and testing of the first low-cost CMOS monolithically-integrated three-axis accelerometer based on thermal convection. A novel single sensor element measures accelerations in all three axes. Differential temperature readout is used in the $\mathrm{X}-\mathrm{Y}$ axis and pseudo-differential mode readout is used for the $\mathrm{Z}$-axis. On-chip, low-noise, analog electronics are used to pre-amplify and condition sensor signals prior to on-chip digital calibration and compensation. This device has a bandwidth of $10 \mathrm{~Hz}$ in all three axes, noise floor of $0.1 \mathrm{mg} / \sqrt{\mathrm{Hz}}$ in the $\mathrm{X}-\mathrm{Y}$ axis and $1.5 \mathrm{mg} / \sqrt{\mathrm{Hz}}$ in the Z-axis. Nonlinearity in the $\mathrm{X}, \mathrm{Y}$, and $\mathrm{Z}$ axes is less than $5 \%$ of full scale. The 3 -axis die is $30 \%$ smaller in area than our current 2 -axis production die and device power remains unchanged at $6 \mathrm{~mW}$.
\end{abstract}

\section{INTRODUCTION}

MEMSIC has been building two axis thermal convection based accelerometers for over ten years ([1], [2], [3]). These sensors have been demonstrated to have high reliability and ultralow manufacturing cost. Since no proof mass is required for this sensor, the thermal bridge may be designed to have very low mass and high resonant frequency (above $50 \mathrm{kHz}$ ), and as a result can survive in-use shock loads above 50,000g. A die image of our twoaxis sensor is shown in Figure 1.

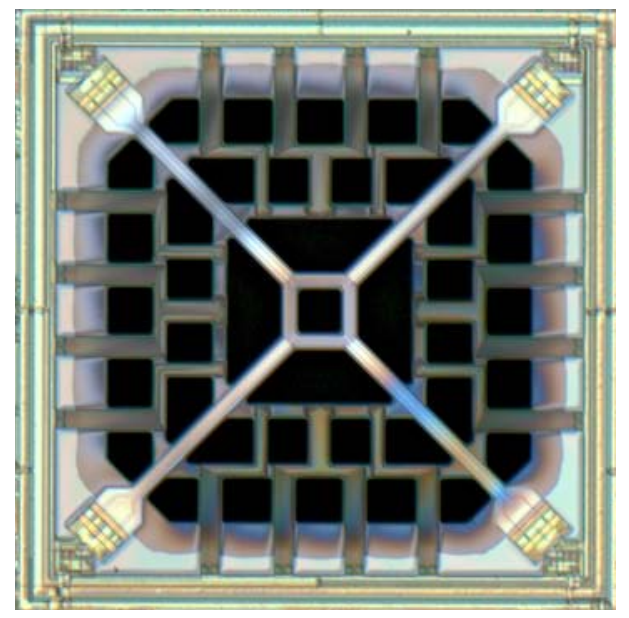

Figure 1: Photograph of a MEMSIC 2-axis sensor element. CMOS circuitry surrounds the sensor on all four sides. The sensor element is approximately $1 \mathrm{~mm}$ by $1 \mathrm{~mm}$ in size.

A heater in the center of the structure sets up a symmetric temperature field (Figure 2) as a result of thermal conduction, which in turn creates a gas density gradient around the structure. Any acceleration in the $\mathrm{X}-\mathrm{Y}$ directions (due to gravity or otherwise) modifies convection currents around the heater that transfer thermal power in the direction of the acceleration and perturb the temperature field asymmetrically. Although these temperature changes are small at the size scales of interest they may be measured differentially using thermopiles on either side of the heater. The convection currents are set up almost instantly with step changes in accelerations, however, the bandwidth of the sensor is limited by the time constant associated with heating or cooling the thermopile elements.

\section{THREE-AXIS ACCELEROMETER}

Ideally, a three dimensional sensing element with thermopiles distributed above and below the heater plane could be fabricated to measure $\mathrm{Z}$-axis accelerations. However, this type of structure is difficult to implement in our standard CMOS-MEMS process. An alternative implementation utilizes the asymmetry of the temperature field in the Z-direction (Figure 2) caused by the cavity below the sensor element. Any Z-axis acceleration would move the isothermal surfaces up or down and modify the common mode temperature of the $\mathrm{X}$ and $\mathrm{Y}$ thermopiles (a symmetric temperature field would produce no common mode signal). The signal must be differentiated from that due to ambient temperature changes. This is achieved using the pseudo-differential readout where a signal proportional to the ambient temperature is subtracted from the sensor common mode signal. It should be pointed out that this technique is inherently less sensitive than a true differential readout and can be more susceptible to drift errors.

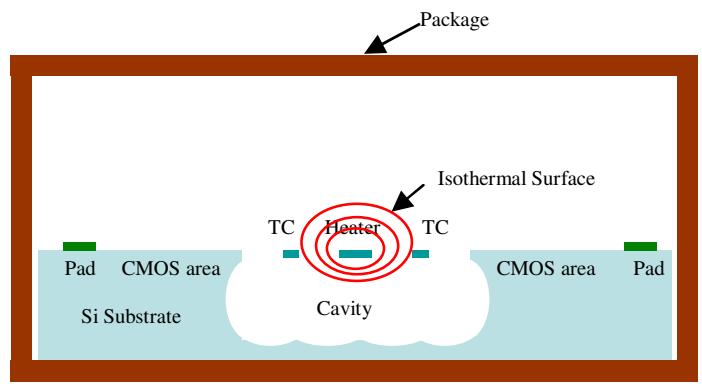

Figure 2: Cross-section view of a thermal accelerometer showing $z$-axis asymmetric temperature field. A cavity under the suspended heater and thermopile is formed by a combination of DRIE and isotropic etches. The cavity depth is approximately 300um. The overall power of the 3-axis device is equal to that of our 2-axis device (approximately $6 \mathrm{~mW}$ ).

The design has been implemented in a 0.25 um CMOS process (Figure 3) that enables implementation of trimming and sophisticated signal processing functions to be moved to the digital domain. Examples include temperature compensation, free fall detection, $\mathrm{I}^{2} \mathrm{C}$ and SPI interfaces. Calibration data is also stored on chip using a one time programming memory (OTP). 


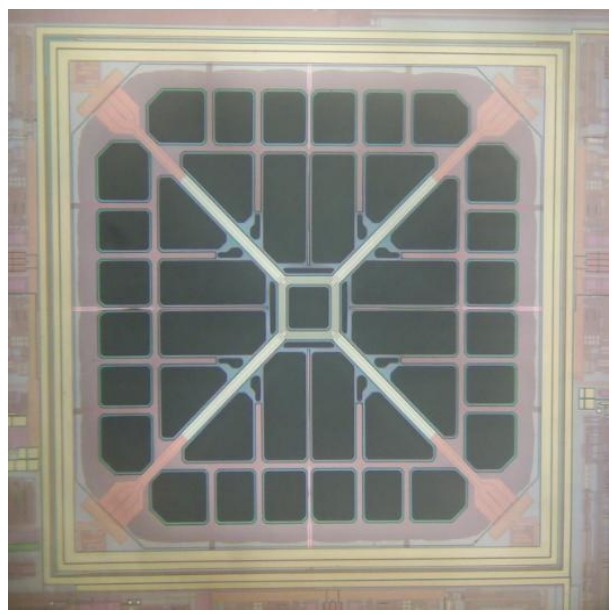

Figure 3: Photograph of a MEMSIC 3-axis sensor element. CMOS circuitry surrounds the sensor on all four sides. The sensor element is identical in size to our 2-axis design, approximately $1 \mathrm{~mm}$ by $1 \mathrm{~mm}$ in size.

\section{EXPERIMENTAL RESULTS}

The Z-axis noise spectral density in the sensor's analog output is shown in Figure 4. X-axis and Y-axis noise is over fifteen times smaller than noise in the z-axis due to higher sensitivity in these directions and approximately equal to that in our existing 2-axis devices. Measurements were taken with an HP 3582A spectrum analyzer.

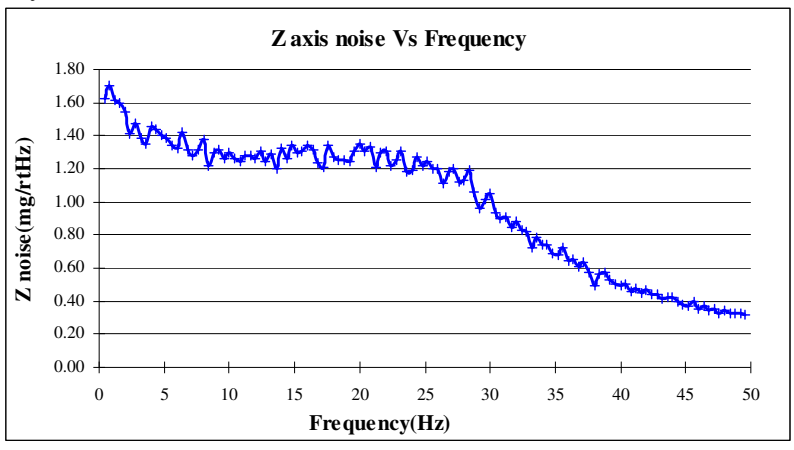

Figure 4: Noise spectral density of the Z-axis signal.

Figure 5 shows the Z-axis output measured through the digitized $\mathrm{I}^{2} \mathrm{C}$ interface. The sensor was rotated by 360 degrees around the $\mathrm{X}$-axis in 6 degree increments. Linearity error was defined as the difference between the measured Z-axis signal (square dots) and a perfect sinusoid (solid line) normalized to $1 \mathrm{~g}$ amplitude. Linearity error was less than 5\% (dashed line with diamonds) after on-chip compensation.

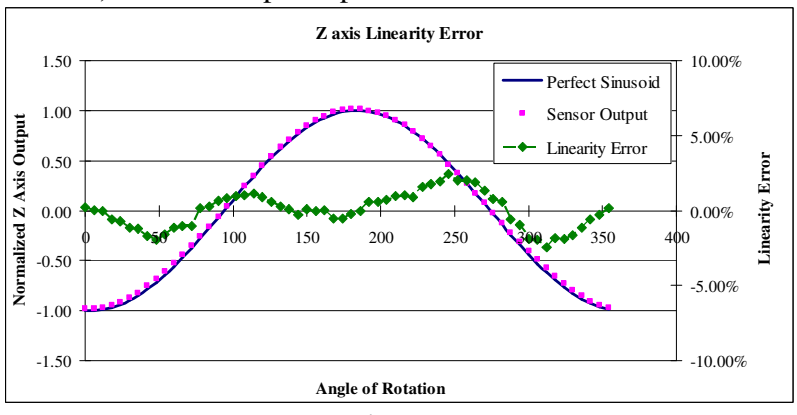

Figure 5: Z-axis linearity measurement.
Figure 6 shows data from a free fall detector that may be used in applications such as hard disk protection. The device is released at $650 \mathrm{~ms}$ from a height of $25 \mathrm{~cm}$ and takes approximately $230 \mathrm{~ms}$ before hitting a table surface. Large accelerations in all three axes are observed upon impact. Free fall is detected within $40 \mathrm{~ms}$ of release, and more than $190 \mathrm{~ms}$ before impact for a $25 \mathrm{~cm}$ drop. When free-fall is detected, a high interrupt signal is generated on an output pin (blue line). A second interrupt signal is observed when the device bounces and begins a second free-fall.

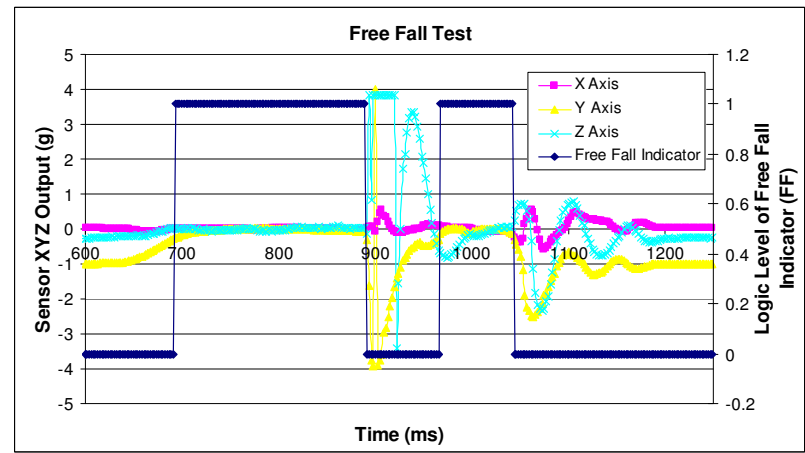

Figure 6: Free fall detection measurement.

\section{CONCLUSION}

We have reported on the first CMOS monolithicallyintegrated three-axis accelerometer based on thermal convection. A novel single sensor element is used to detect accelerations in all three axes. Differential mode sensing is used for $\mathrm{X}$ and $\mathrm{Y}$ axes and a pseudo-differential mode is used for the Z-axis. The device is fabricated in a $0.25 \mathrm{um}$ CMOS process with on-chip amplification, digital signal processing and calibration. Total die area is reduced by $30 \%$ from our 2-axis designs while keeping overall power unchanged.

\section{REFERENCES}

[1] A. M. Leung, J. Jones, E. Czyzewska, J. Chen, B. Woods 1998 Micromachined accelerometer based on convection heat transfer. Proc. 11th IEEE Int. Workshop Micro Electro Mechanical Systems (MEMS '98), Heidelberg, Germany, pp. 627-630

[2] Y. Zhao, P. Brokaw, M. E. Rebeschini, A. M. Leung, G. P. Pucci, A. Dribinsky "Thermal convection accelerometer with closed-loop heater control", U.S. Patent 6795752, issued 2004

[3] Y. Zhao, Y. Hua, "Method of etching a deep trench in a substrate and method of fabricating", U.S. Patent 6712983, issued 2004 